\title{
Adjustment for physical activity in studies of sedentary behaviour
}

Andrew Page ${ }^{1 *}$, Geeske Peeters ${ }^{2}$ and Dafna Merom ${ }^{3}$

\begin{abstract}
Sedentary behaviour (too much sitting, as distinct from too little exercise) has emerged as a potentially significant public health issue. Analytically, researchers have reported 'independent' associations between sedentary behaviour (SB) and a number of health outcomes by adjusting for physical activity (PA) (and other confounders), and conclude that $\mathrm{SB}$ is associated with the outcome even in those who are physically active. However, the logical rationale for why adjustments for PA are required is often not delineated, and as a consequence, PA has been conceptualised as a confounder, an intermediary, and an effect measure modifier-sometimes simultaneously_in studies of SB and health outcomes. This paper discusses the analytical assumptions underlying adjustment for PA in studies of SB and a given outcome, and considers the implications for associations between SB and health.
\end{abstract}

Keywords: Sedentary behaviour, Physical activity, Methods, Measurement, Collinearity

\section{Background}

In the past decade physical activity and public health research has shifted its focus to the harms of 'sedentary behaviour' (SB). Sedentary behaviour (too much sitting, as distinct from too little physical activity) has emerged as a potentially significant public health issue, given its associations with morbidity [1], and all-cause and cause-specific mortality [2-5]. 'Too much sitting' has been described as an independent risk factor of chronic disease, irrespective of amounts of moderate to vigorous physical activity (MVPA), possibly due to differential effects on health outcomes. SB and physical activity relate differently to health outcomes and via distinct biological mechanisms, $[6,7]$ and it is possible to have both high SB and MVPA in a 24-h period.

The protective effect of MVPA on health outcomes relates to improvements in cardio-respiratory fitness through increased oxygen supply to the myocardium and improved myocardial contraction [8], as well as lower blood pressure [9], improved lipoprotein profile [10], and increased insulin sensitivity [11]. The

\footnotetext{
*Correspondence: a.page@uws.edu.au

${ }^{1}$ Centre for Health Research, School of Medicine, University of Western

Sydney, Campbelltown, Australia

Full list of author information is available at the end of the article
}

main biological mechanism proposed for SB relates to cardio-metabolic changes associated with decreased lipoprotein lipase activity (associated with increases in plasma triglycerides and decreases in HDL-cholesterol), which are associated with coronary heart disease, type II diabetes and obesity [12]. It is hypothesised that chronic exposure to SB reduces skeletal muscle contractile activity which evokes a process of suppressing the amount of capillary lipoprotein lipase in the muscle [13].

However, the way in which these two exposures are specified in analyses is often confused, and does not necessarily relate to clear biological mechanisms. Physical activity (commonly operationalized as achieving the recommended MVPA for health) has been considered as a confounder, intermediary, and effect measure modifier, sometimes simultaneously [1] in studies of sedentary behaviour and health outcomes. The logical rationale for why adjustment for physical activity is required is often not clearly articulated, nor is there always a clear delineation of the temporal ordering of measurement periods of SB and PA. This paper discusses the analytical assumptions underlying adjustment for PA in studies of SB and a given outcome, and considers the implications for associations between SB and health outcomes. 


\section{Causal assumptions underlie simultaneous adjustment}

Analytically, researchers obtain 'independent' associations between sedentary behaviour (SB) and a given outcome by adjusting for physical activity (PA), and conclude that $\mathrm{SB}$ is associated with the outcome even in those who are physically active (that is, achieving recommended MVPA). By including PA and SB simultaneously in a statistical model, there is an implied causal assumption, illustrated by the directed acyclic graphs in Figures 1 and 2. If PA is considered a common cause (confounder) of $\mathrm{SB}$ and the outcome (D), the implication is that PA causes SB (Figure 1a), and adjustment is necessary. There may also be an exogenous variable $(\mathrm{C})$, such as injury or disability status, which is a common cause of both PA and $\mathrm{SB}$, and where the causal direction between PA and SB is unknown. In this case adjustment for PA is necessary if $C$ is unmeasured and PA is not collinear with $\mathrm{SB}$ (Figure 1b).

If $\mathrm{PA}$ is considered an intermediary between $\mathrm{SB}$ and $\mathrm{D}$, then the implication is that PA is an effect of $\mathrm{SB}$, and adjustment is conducted to demonstrate the extent of mediation, and the 'independent' effect of SB on D (Figure 2a). This latter adjustment is problematic-as for all mediation analyses using conditional models-as adjustment for PA as an intermediary induces an association between $\mathrm{SB}$ and unmeasured confounders $(\mathrm{U})$

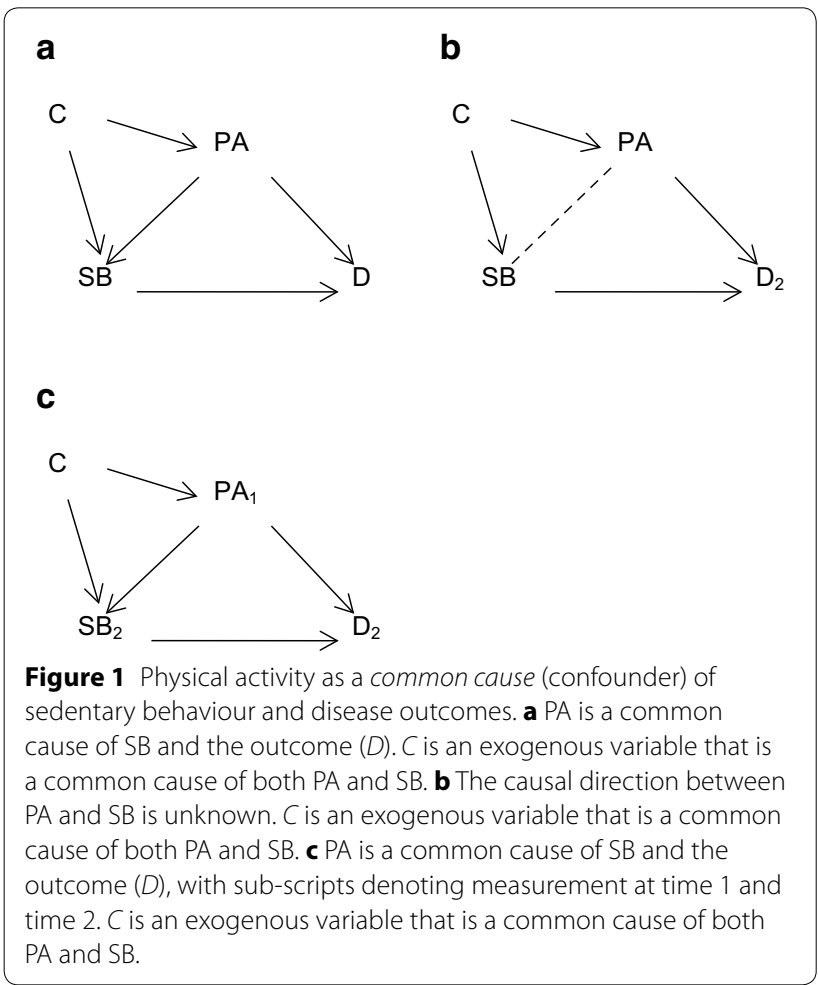

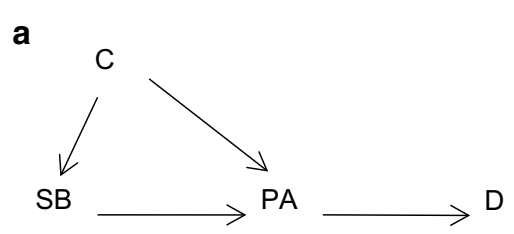

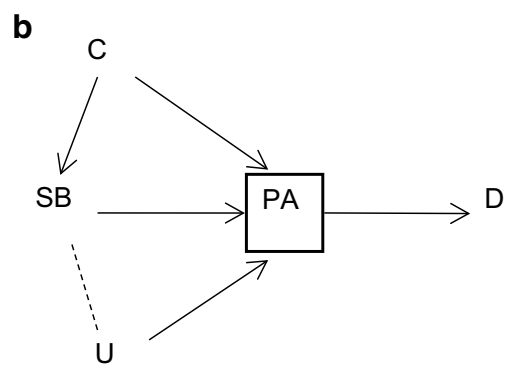

Figure 2 Physical activity as an intermediary between sedentary behaviour and disease outcomes. $\mathbf{a}$ PA is an intermediary between SB and D. b Adjustment for PA as an intermediary induces an association between SB and unmeasured confounders $(U)$ by conditioning on the common effect PA.

by conditioning on the common effect of PA (Figure 2b) (known as collider stratification bias) [14, 15]. Unless there are explicitly measured temporal relationships between PA and SB (Figure 1c), where people do more (or less) MVPA at time 1 and therefore become more (or less) sedentary at time 2, then conceptualising PA as a confounder or intermediary assumes a causal direction that may not be warranted based on the putative aetiological mechanisms by which PA and SB affect health outcomes.

Additionally, studies usually do not explicitly delineate the temporal ordering of SB and PA measures, and do not analytically address the inter-dependence of $\mathrm{SB}$ and PA across time: SB is a time-dependent exposure that is analysed in the presence of the time-dependent co-variate of PA. In this context, PA can be considered as both a confounder and an intermediary, where PA is an effect of SB but is also a common cause of SB and D (Figure 3). If conceptualising PA as a time-dependent co-variate of

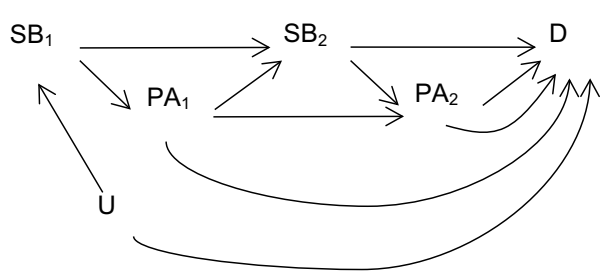

Figure 3 Physical activity as a confounder and intermediary between sedentary behaviour and disease outcomes. Sub-scripts denote measurement at time 1 and time 2 . 
SB (a time-dependent exposure) is a more appropriate reflection of the putative aetiological mechanisms by which PA and SB affect health outcomes, then marginal structural models (MSMs) [16] can be used to estimate the 'independent' effects of SB on a given health outcome. MSMs provide a solution to the collider stratification bias inherent in conditional approaches to adjustment for PA in studies of SB and a given outcome (Figure 2). MSMs derive inverse probability weights to re-weight a dataset to reflect the probability of a given level of PA based on observed level of SB. This approach allows unbiased estimation of the marginal association between SB and D, adjusting for PA.

\section{Biological interaction, statistical interaction, or collinearity}

An alternative conceptualisation, and the reason for why SB might be considered separately to PA, is that SB has a different aetiological pathway to $\mathrm{D}$, and that the presence of some level of PA or SB affects the level of the other. That is, the relationship is one of effect measure modification due to the interaction between plausible biological mechanisms (Figure 4). For example, perhaps reductions in lipoprotein lipase (LPL) activity affect oxidative muscle fibres in response to SB (with subsequent deleterious effects on metabolic health), whereas increases in LPL affect glycolytic fibres in response to PA (with subsequent beneficial effects on metabolic health) [6].

A number of observational studies have considered interactions between $\mathrm{PA}$ and $\mathrm{SB}[5,17,18]$, to show that the magnitude of the association between SB and D differs within levels of PA in associations with disease outcomes. Conceptualising PA as an effect measure modifier of an SB-D association may be a more appropriate causal model, if PA does not cause SB and vice versa. If there are plausible and independent biological mechanisms for $\mathrm{PA}$ and $\mathrm{SB}$, then this may be the most appropriate way of conceptualising the separate or synergistic effects of SB and PA on health outcomes. However, if SB and PA are two sides of the same coin (that is, the energy expenditure continuum), then testing effect measure modification

\section{a}

SB

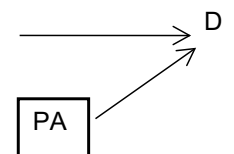

\section{b}

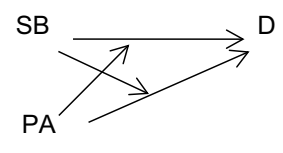

Figure 4 Physical activity as an effect measure modifier of associations between sedentary behaviour and disease outcomes. a The association between SB and D differs in magnitude within strata of PA. $\mathbf{b}$ Model of effect measure modification proposed by Weinberg [20]. here may be analogous to testing effect measure modification by number of cigarette packs smoked per day in the association between smoking status and lung cancer.

Figure $4 \mathrm{a}$ shows a causal graph that is compatible with direct effect measure modification [19], and which implies that within strata of PA (for example, sufficient or insufficient MVPA), the association between SB and $\mathrm{D}$ differs in magnitude. Some authors have criticised this specification of effect measure modification using causal graphs, as it does not sufficiently capture the myriad ways in which an effect measure modifier may affect an exposure-disease association [20]. Weinberg [20] illustrates the limited way in which causal graphs can specify effect measure modification by proposing a range of alternative models for known biological and environmental interactions, which are not strictly 'directed acyclic graphs' in that not all vertices emanating from an 'ancestor' terminate at a 'descendant' variable, which is a basic condition [21].

However, one model of effect measure modification proposed by Weinberg [20] (Figure 4b), is consistent with the inherent inter-dependence between SB and PA and how SB and PA may independently affect disease outcomes. In this model, the expectation $\mathrm{E}(\mathrm{D} \mid \mathrm{SB}, \mathrm{PA})$ is not constant in SB for any fixed value of PA, and is not constant in PA for any fixed value of SB. Neither SB nor PA is a cause of the other, however on a given effect measure modification scale for some pair of $\mathrm{SB}$ values $\left(\mathrm{SB}_{1}\right.$ and $\left.\mathrm{SB}_{0}\right)$ and some pair of $\mathrm{PA}$ values $\left(\mathrm{PA}_{1}\right.$ and $\left.\mathrm{PA}_{0}\right)$ then there is effect measure modification: $\mathrm{E}\left(\mathrm{D} \mid \mathrm{SB}_{1}, \mathrm{PA}_{1}\right)-\mathrm{E}\left(\mathrm{D} \mid \mathrm{SB}_{0}\right.$, $\left.\left.\mathrm{PA}_{0}\right) \neq \mathrm{E}\left(\mathrm{D} \mid \mathrm{SB}_{1}, \mathrm{PA}_{0}\right)-\mathrm{E}\left(\mathrm{D} \mid \mathrm{SB}_{0}, \mathrm{PA}_{0}\right)\right]+\mathrm{E}\left(\mathrm{D} \mid \mathrm{SB}_{0}, \mathrm{PA}_{1}\right)-$ $\mathrm{E}\left(\mathrm{D} \mid \mathrm{SB}_{0}, \mathrm{PA}_{0}\right)$. Few studies consider statistical interaction between PA and SB on the additive scale [3,22] which is the most appropriate scale to examine joint effects of plausible biological mechanisms.

It is also important to make the distinction between statistical and biological interaction. Statistical interaction is dependent on an arbitrary choice of scale, whereas biological interaction exists or does not, and can be investigated in joint effects analyses [23]. However, this distinction between statistical and biological interaction is contestable, and is inherently difficult to estimate even with defined biological mechanisms [24, 25]. In instances where PA is specified as an effect measure modifier of $\mathrm{SB}$ and $\mathrm{D}$, it is not clear whether this reflects statistical interaction or biological interaction given the often selfreported nature of exposure measurement and the inherent collinearity between SB and PA categorised over a $24 \mathrm{~h}$ time period [26]. All dimensions of activity during a 24-h period (i.e. MVPA, light intensity PA, standing, $\mathrm{SB}$, and sleep) may be associated with health outcomes, however, simultaneous adjustment of these dimensions of activity and/or assessment of substitution of effects (of 
$\mathrm{SB}$ to PA or vice versa) is problematic given this collinearity [26].

In studies where self-reported measures of $\mathrm{PA}$ and $\mathrm{SB}$ are used, this inherent collinearity may not be explicit, given that PA measures are often restricted to MVPA (often in one activity domain) ensuring a notionally nonidentifiable relationship between PA and SB (although light intensity PA and total PA can also be included in analyses as residual confounders). If time spent in all intensity levels is measured using, for example, an accelerometer for a given 24-h period, then there is an explicitly identifiable relationship between PA and SB measures.

\section{Should any adjustment be made?}

If PA is not a common cause of SB and D, and is not an effect of SB on the causal pathway to D (an intermediary), then adjustment for PA in models of SB and D is not required, in either standard conditional models or marginal structural models. If there is evidence of statistical interaction between SB and PA then further research should establish whether this interaction is an artefact of the arbitrary selection of statistical scale, or whether this represents biologically distinct causal pathways for SB and PA associated with $\mathrm{D}$. If SB is simply the complementary (and identifiable) component of PA in the total $24 \mathrm{~h}$ period of energy expenditure, and not dependent on the presence of the other (and not an effect measure modifier), then it is unclear as to why analyses would need to consider both PA and SB simultaneously at all.

\section{Authors' contributions}

AP conceived and drafted the manuscript. GP and DM provided critical revisions of the manuscript for important intellectual content. All authors read and approved the final manuscript.

\section{Author details \\ ${ }^{1}$ Centre for Health Research, School of Medicine, University of Western Sydney, Campbelltown, Australia. ${ }^{2}$ School of Public Health, The University of Queensland, Brisbane, Australia. ${ }^{3}$ School of Science and Health, University of Western Sydney, Campbelltown, Australia.}

\section{Compliance with ethical guidelines}

\section{Competing interests}

The authors declare that they have no competing interests.

Received: 26 February 2015 Accepted: 30 June 2015

Published online: 09 July 2015

\section{References}

1. Thorp AA, Owen N, Neuhaus M, Dunstan DW (2011) Sedentary behaviors and subsequent health outcomes in adults a systematic review of longitudinal studies, 1996-2011. Am J Prev Med 41(2):207-215. doi:10.1016/j. amepre.2011.05.004
2. Chau JY, Grunseit AC, Chey T, Stamatakis E, Brown WJ, Matthews CE et al (2013) Daily sitting time and all-cause mortality: a meta-analysis. PLoS One 8(11):e80000

3. Katzmarzyk PT, Church TS, Craig CL, Bouchard C (2009) Sitting time and mortality from all causes, cardiovascular disease, and cancer. Med Sci Sports Exerc 41(5):998-1005. doi:10.1249/MSS.0b013e3181930355

4. Koster A, Caserotti P, Patel KV, Matthews CE, Berrigan D, Van Domelen DR et al (2012) Association of sedentary time with mortality independent of moderate to vigorous physical activity. PLoS One 7(6):e37696. doi:10.1371/journal.pone.0037696

5. Matthews CE, George SM, Moore SC, Bowles HR, Blair A, Park Y et al (2012) Amount of time spent in sedentary behaviors and cause-specific mortality in US adults. Am J Clin Nutr 95(2):437-445. doi:10.3945/ ajcn.111.019620

6. Tremblay MS, Colley RC, Saunders TJ, Healy GN, Owen N (2010) Physiological and health implications of a sedentary lifestyle. Appl Physiol Nutr Metab 35(6):725-740

7. Brown WJ, Bauman AE, Owen N (2009) Stand up, sit down, keep moving: turning circles in physical activity research? Br J Sports Med 43(2):86-88

8. Paffenbarger RS, Lee IM (1996) Exercise and fitness. In: Manson J, Ridker P, Gaziano J, Hennekens C (eds) Prevention of myocardial infraction. Oxford University Press, New York

9. Kelley GA, Kelley KS, Tran ZV (2001) Walking and resting blood pressure in adults: a meta-analysis. Prev Med 33(2):120-127

10. Kelley GA, Kelley KS, Tran ZV (2004) Walking, lipids, and lipoproteins: a meta-analysis of randomized controlled trials. Prev Med 38(5):651-661

11. Knowler WC, Barrett-Connor E, Fowler SE, Hamman RF, Lachin JM, Walker EA et al (2002) Reduction in the incidence of type 2 diabetes with lifestyle intervention or metformin. N Engl J Med 346(6):393

12. Hamilton MT, Hamilton DG, Zderic TW (2007) Role of low energy expenditure and sitting in obesity, metabolic syndrome, type 2 diabetes, and cardiovascular disease. Diabetes 56(11):2655-2667

13. Hamilton MT, Hamilton DG, Zderic TW (2004) Exercise physiology versus inactivity physiology: an essential concept for understanding lipoprotein lipase regulation. Exerc Sport Sci Rev 32(4):161

14. Cole SR, Hernan MA (2002) Fallibility in estimating direct effects. Int J Epidemiol 31(1):163-165

15. Cole SR, Platt RW, Schisterman EF, Chu H, Westreich D, Richardson D et al (2010) Illustrating bias due to conditioning on a collider. Int J Epidemiol 39(2):417-420. doi:10.1093/ije/dyp334

16. Robins JM, Hernan MA, Brumback B (2000) Marginal structural models and causal inference in epidemiology. Epidemiology 11(5):550-560

17. Patel AV, Bernstein L, Deka A, Feigelson HS, Campbell PT, Gapstur SM et al (2010) Leisure time spent sitting in relation to total mortality in a prospective cohort of US adults. Am J Epidemiol 172(4):419-429. doi:10.1093/aje/kwq155

18. Pavey TG, Peeters GG, Brown WJ (2015) Sitting-time and 9-year all-cause mortality in older women. Br J Sports Med 49(2):95-99

19. VanderWeele TJ, Robins JM (2007) Four types of effect modification: a classification based on directed acyclic graphs. Epidemiology 18(5):561-568

20. Weinberg CR (2007) Can DAGs clarify effect modification? Epidemiology 18(5):569-572

21. Greenland S, Pearl J, Robins JM (1999) Causal diagrams for epidemiologic research. Epidemiology 10(1):37-48

22. Seguin R, Buchner DM, Liu J, Allison M, Manini T, Wang C-Y et al (2014) Sedentary behavior and mortality in older women: the women's health initiative. Am J Prev Med 46(2):122-135

23. Greenland S, Lash T, Rothman K (2008) Concepts of interaction. In: Rothman K, Greenland S, Lash T (eds) Modern epidemiology. Lippincott Williams and Wilkins, Philadelphia, pp 71-86

24. Greenland S (2009) Interactions in epidemiology: relevance, identification, and estimation. Epidemiology 20(1):14-17

25. Lawlor DA (2011) Biological interaction: time to drop the term? Epidemiology 22(2):148-150

26. Pedišić $Z ̌ Z$ (2014) Measurement issues and poor adjustments for physical activity and sleep undermine sedentary behaviour research — the focus should shift to the balance between sleep, sedentary behaviour, standing and activity. Kinesiology 46:135-146 